\title{
Gerencia estratégica para la gestión de personas del sector minero de Venezuela, Colombia y Chile
}

\author{
Reynier I. Ramírez ${ }^{(1)}$, Nelson D. Lay ${ }^{(2)}$ y Harold B. Sukier ${ }^{(1)}$ \\ (1) Universidad de la Costa, Dpto. de Ciencias Empresariales, Atlántico - Colombia. \\ (correo-e: rramirez13@cuc.edu.co; hsukier@cuc.edu.co) \\ (2) Universidad Andrés Bello, Escuela de Psicología, Viña del Mar - Chile. \\ (correo-e: nelsonlay@gmail.com)
}

Recibido Abr. 21, 2019; Aceptado Jun. 24, 2019; Versión final Jul. 31, 2019, Publicado Feb. 2020

\begin{abstract}
Resumen
Se analiza las etapas de la gerencia estratégica para la gestión de personas del sector minero de Venezuela, Colombia y Chile. Se usa una investigación cuantitativa, de tipo descriptivo, diseño no experimental transversal. Se usa la técnica de la encuesta mediante un cuestionario de nueve ítems, aplicado a 55 gerentes, adscritos a cada vicepresidencia de las empresas Carbones del Guasare, SA, Complejo Carbonífero de Cerrejón y Mina Invierno. La técnica de análisis fue descriptiva, de varianza, regresión lineal y de tipo factorial. Los resultados evidenciaron inexistencia de diferencias significativas entre las etapas de la gerencia estratégica, considerada moderada. El estudio mostró que la gerencia estratégica para la gestión de personas potencia ventajas competitivas sostenibles como rasgo distintivo en el sector real, convirtiéndose el colaborador en generador de valor estratégico por sus competencias en la organización, respondiendo a las exigencias del entorno.
\end{abstract}

\section{Management strategy for people management in the mining sector of Venezuela, Colombia and Chile}

\begin{abstract}
The stages of management strategic for the management of people of the mining sector of Venezuela, Colombia and Chile are analyzed. The research is of quantitative type, descriptive, and non-experimental cross-sectional design. The technique of the survey through a nine-item questionnaire, is used and applied to 55 managers, attached to each vice presidency of the companies Carbones del Guasare, SA, Carbonifero Cerrejon and Winter mine. The technique of analysis was descriptive, variance, linear regression and of factorial type. The results showed lack of significant differences between the stages of management strategic, considered to be moderate. The study showed that people management strategic management enhances sustainable competitive advantages as a distinctive feature in the real sector, becoming partner in generator of strategic value for their competence in the Organization, responding to the demands of the environment.
\end{abstract}

Keywords: strategic management; people management; formulation of strategies; evaluation of strategies 


\section{INTRODUCCIÓN}

La gerencia estratégica ha tenido soportes transdisciplinarios para el adecuado manejo de la gestión de personas que involucra la organización como centros de estudios, ante las competencias del ser humano como activo intangible generador de valor en el mercado emergente, todo esto ha pasado por etapas, a través de la historia, escuelas, disciplinas y enfoques, consolidándose en las organizaciones como un factor que contribuye notoriamente al logro sistémico de las metas corporativas, donde lo integral y transdisciplinar, guarda un lugar irremplazable en el talento humano, y por tanto en su accionar (Ramírez et al.,2019). En Latino América la gerencia estratégica para la gestión de personas, se apreció como un modo de trabajar con el personal, apoyándose en la valoración de funciones sencillas, progresando hasta convertirse en un instrumento sólido de planeación, dirección y control (Armstrong. 2016; Wheelen et al., 2018). En países como Colombia, Argentina, Brasil, Chile, Ecuador, Perú y Venezuela, se ha implementado la gestión del talento humano en su estrategia organizacional, acompañando al personal, innovando indicadores de compensación y políticas retentivas, estimulando un resultado motivacional hacia el cumplimiento de metas, apostando a lo estratégico y creativo (Pérez et al., 2012).

Las empresas para consolidar su razón de ser, necesitan personal de alto potencial, con miras hacia el futuro. Debido a la situación demográfica, social y económica en Europa, el mercado laboral está reflejando insipientes avances en la calidad de gestión humana, inspirando a la investigación y tratamiento programado del talento humano, es decir, los colaboradores talentosos, (Boštjančič y Slana, 2018; Ling et al., 2018). Las organizaciones son conscientes que sólo aquellas que reconocen esta área como fundamental, tendrán éxito en la guerra de talentos. Por tal motivo, múltiples referentes conceptuales sobre la gerencia estratégica para la gestión de personas, en estos últimos años se ha convertido en una línea de investigación en numerosas empresas, trascendentales en la praxis del talento humano en las organizaciones. Reflejando enfoques: estrategia global o corporativa, de unidades de negocios, posibilitando la dirección estratégica y cambio, y sus procesos fundamentales. Para Dirani (2018), la gestión de gente, pasa por tiempos inestables. Las organizaciones operan en un entorno diverso proyectado por la visión de un gobierno inteligente y políticas prácticas que permitan etiquetarse como un destino de imán de talento.

Actualmente en las empresas Carbones del Guasare, S.A (Venezuela), Complejo Carbonífero de Cerrejón (Colombia) y Mina Invierno (Chile), se están adaptando a necesidades funcionales específicas y requerimientos del entorno, considerando la situación del país de acuerdo a sus factores sociocultural, políticos y económicos, estableciendo cambios en su estrategia organizacional, generando acciones en oportunidades improvisadas, impactando laboralmente al personal, redes empresariales, clúster y stakeholder. Todo esto a causa de precipitados cambios que sufre cada organización en su país, falta de iniciativas de apostar a lo nuevo, y ausencia de garantías de creer en la gente. Para Sapiro, (2017) y Boštjančič y Slana (2018), estas situaciones traen como consecuencia aplicaciones empresariales erróneas, clima organizacional inestables, amplias brechas de conocimientos entre los trabajadores, esfuerzos individuales de trabajo que se pueden simplificar, tomas de decisiones desarticuladas, bajo desempeño individual y colectivo del colaborador, limitando el alcance de metas con resultados óptimos, impactando social como económicamente las prácticas laborales, trascendiendo negativamente hasta en la vida del personal. Ante este escenario, crecen las expectativas en las empresas estudiadas de valorar la gerencia estratégica para la gestión de personas, definiendo objetivos que direccionarán su evolución organizacional, buscando consolidarse en el mercado minero, a fin de ser referentes internacionales, con un alto desempeño organizacional. Ante estas consideraciones, se formula la siguiente interrogante: ¿Cómo son las etapas de la gerencia estratégica para la gestión de personas del sector minero de Venezuela, Colombia y Chile?

Al desmeritar la gerencia estratégica para la gestión de personas del sector minero de Venezuela, Colombia y Chile, se dejaría de conservar una orientación táctica general de la empresa ante los requerimientos del sector real, desvirtuando la aceptación, empleo, indemnización, desarrollo, contención y dirección del personal, con iniciativas que incrementen estratégicamente el aprovechamiento productivo de la empresa, propiciado un valor único en estas, redundando en un posicionamiento competitivo en el mercado. Por tal motivo, en el estudio se analizan las etapas de la gerencia estratégica para la gestión de personas del sector minero de Venezuela, Colombia y Chile, a fin de generar aportes que impacten positivamente los indicadores de gestión corporativos, útiles a la gerencia y fortalezca el estilo de acompañar a la gente como socios estratégicos de la organización, respondiendo eficazmente a las necesidades estructural-global de las empresas en un mundo dinámico, inestable, agresivo y competitivo, cada vez más habido de talento humano innovador, resolutivo, liderente, dispuesto a desaprender para aprender y emprender. 
La nueva era del conocimiento, global, intangible y artificial, ha permitido investigar el por qué algunas organizaciones son exitosas y otras luchan por mantenerse en el mercado. Por tal motivo, expertos en gerencia estratégica para la gestión de personas, han concentrado su atención en estos últimos años sobre esta variable. Esto ha afectado notoriamente el comportamiento del mercado ante la oferta y demanda, impactando significativamente la rentabilidad corporativa y de allí su supervivencia, originando que las empresas consideren la formulación, ejecución y evaluación de estrategias, como indicadores claves para implementar ventajas competitivas en el mundo emergente.

La gerencia estratégica define la formulación, ejecución y evaluación (Fayol,1971; Serna, 2008), de acciones que permitirán a la organización el logro de sus objetivos (Delgado, 2011; Cuesta, 2015), impactando sistémicamente las acciones ejecutadas por el talento humano (Mendoza, 2015; Fuentes y Cordón, 2016). Organiza cualitativa y cuantitativamente la toma de decisiones efectivas (Chiavenato y Sapiro, 2017), minimizando la incertidumbre ante cambios bruscos e inestables del ambiente, de acuerdo a criterios y análisis pertinentes (Dirani 2018; Fred 2018), con la capacidad de influir y ejercer el control en su medio natural, sincronizando activa y efectivamente las áreas funcionales del trabajo, generando valor agregado a la cadena socio productiva empresarial (McDonnell et al., 2017; Boštjančič y Slana, 2018).

La gerencia estratégica, es el arte y ciencia de proponer, desarrollar y evaluar decisiones multidisciplinarias, alcanzando el objetivo empresarial, integrando la administración, marketing, finanzas, contabilidad, producción, investigación y desarrollo, y sistemas de información, logrando el éxito organizacional. Describe y desarrolla las tácticas, analizando el entorno empresarial, ventajas competitivas y aprovechamiento de las oportunidades para defenderse de las amenazas (Fred, 2018; Meyer y Xin, 2018). Para León (2011), es el instrumento que establece indicadores de logros y control, identificando procesos críticos dentro de la gestión, enfoques, y demás áreas que tengan concordancia con la misión, visión y objetivos establecidos. Expresado de otra forma, es la materia prima o insumo fundamental para aplicar la planeación estratégica, táctica y operativa, garantizando alcanzar el lugar propuesto, contribuyendo a la estructura y modelo de gestión humana requerida.

En la integración de la gerencia estratégica para la gestión del talento humano se crea la capacidad que les confiere a las empresas, una ventaja competitiva sostenible como rasgo distintivo ante la competencia basada en las personas. Existe una necesidad fundamental de desarrollar en estos las habilidades necesarias para trabajar de forma eficiente con cualidades diferenciadoras, dotándole de responsabilidad para que se consideren auténticos promotores del éxito de la empresa. Ante tal premisa, el talento humano se convierte en el centro de apoyo que integra las capacidades, conocimientos y habilidades, que conlleva la consecución de una ventaja competitiva sostenible, siendo el mecanismo de vinculación de los demás recursos en la empresa. Fuentes y Cordón (2016), aluden que es un sistema de dirección que sirve para diseñar y ejecutar estrategias, respondiendo a las exigencias del entorno, integrado por la formulación, ejecución y evaluación de estrategias. Para Mendoza (2015), la integración de la gerencia estratégica para la gestión de personas es la administración postmoderna, determinada por tácticas funcionales, consolidando actividades operativas- empresariales, como el: mercadeo, producción, finanzas y recursos humanos, enfrentando decisiones estratégicas gerenciales con límites de plazo significativos.

La formulación de estrategia, es un proceso de identificación de oportunidades y amenazas en la empresa, que precisa fortalezas y debilidades (Fred, 2018), comprenden actividades formales e informales (Fuentes y Cordón, 2016), socialización de necesidades y requerimiento (Ramírez y Hugueth, 2017), determinando objetivos, diferenciando el alcance y magnitud de las tácticas, direccionando condiciones futuras basadas en información del presente, mediados por los principios corporativos, estrategias, modelo de negocio, proyectos y cronograma de gestiones tácticas, describiendo nuevos negocios, incursionando mercados internacionales, formando alianzas y evitar decisiones hostiles (Duran et al., 2017; Mendoza, 2015).

La ejecución de estrategias, es el desarrollo de acciones que intervienen en las actividades empresariales entre las dependencias y el entorno, generando procesos competitivos, considerando recursos en las tácticas gerenciales, incluyéndolas en la formación de equipos de alto desempeño en áreas medulares (Delgado, 2011; Fred, 2018). Es la puesta en práctica de cada acción, reflejados en los planes estratégicos, de acuerdo a decisiones óptimas para enfrentar cambios del entorno. (Duran et al., 2017; Fuentes y Cordón, 2016; Mendoza, 2015). Por su parte, la evaluación de estrategias, es el proceso de análisis externo e interno, respondiendo a las modificaciones del entorno, originando decisiones en la empresa, posibilitando ventajas competitivas, direccionando oportunidades, amenazas, fortalezas, y debilidades del sector, alimentando criterios de control, comparando los resultados obtenidos con los deseados, se analizan los efectos en el área, especificando las consecuencias del sistema (Delgado, 2011; Fred, 2018). Para Mendoza (2015), es el análisis de estrategias que permitirán integrar recursos actuales hasta que surja sinergia, evaluando tácticas de diversificación por efectos semejantes de los recursos. 
Al considerar los referentes conceptuales clásicos y actuales de corrientes principales de la gerencia estratégica para la gestión de personas, de acuerdo a Fayol (1971), Serna (2008), Cuesta (2015), Chiavenato y Sapiro, (2017), McDonnell et al., (2017), Dirani (2018), Fred (2018) y Boštjančič y Slana (2018) en la tabla 1 se muestran argumentos definitorios, como actividades sistémicas basadas en información del presente, que permiten identificar fortalezas para utilizarse como elemento distintivo, aprovechar oportunidades, disminuir debilidades, evitando amenazas en un mercado emergente y competitivo. Se busca determinar el alcance y magnitud de las tácticas, incursionando en nuevos negocios, formando equipos de alto desempeño en áreas medulares para enfrentar cambios del entorno, con criterios de control, al comparar resultados, valorando efectos estratégicos en el área de labor, especificando consecuencias, para evitar cualquier costo de error.

Tabla 1: Elementos conceptuales de los indicadores de la gerencia estratégica para la gestión de personas

\begin{tabular}{|c|c|}
\hline $\begin{array}{c}\text { Indicadores de la gerencia estratégica } \\
\text { para la gestión de personas }\end{array}$ & Elementos conceptuales \\
\hline Formulación de estrategias. & $\begin{array}{l}\text { Comprende actividades formales e informales, direccionando } \\
\text { condiciones futuras basadas en información del presente. } \\
\text { Identifica fortalezas, debilidades, oportunidades y amenazas } \\
\text { corporativas. } \\
\text { Diferencia alcance y magnitud de las tácticas. } \\
\text { Describe nuevos negocios, incursionando en mercados } \\
\text { internacionales, formando alianzas. }\end{array}$ \\
\hline Ejecución de estrategias. & $\begin{array}{l}\text { Desarrolla actividades empresariales en las dependencias y } \\
\text { entorno. } \\
\text { Genera procesos competitivos. } \\
\text { Considera recursos en las tácticas gerenciales, incluyéndolas en } \\
\text { la formación de equipos de alto desempeño en áreas medulares. } \\
\text { Potencia sus fortalezas y prevé oportunidades del sector real. }\end{array}$ \\
\hline Evaluación de estrategias. & $\begin{array}{l}\text { Evalúa cada acción, reflejados en el análisis de los planes } \\
\text { estratégicos para enfrentar cambios del entorno. } \\
\text { Permite el análisis externo e interno de las decisiones tomadas. } \\
\text { Disminuye debilidades del sistema, e identifica amenazas para } \\
\text { evitar decisiones aisladas. } \\
\text { Alimenta criterios de control, comparando resultados con los } \\
\text { deseados. } \\
\text { Analiza efectos estratégicos en el área de labor, especificando } \\
\text { consecuencias. }\end{array}$ \\
\hline
\end{tabular}

\section{METODOLOGÍA}

El paradigma fue positivista de carácter cuantitativo, de tipo descriptivo, diseño no experimental transeccional, incluyendo actividades sugeridas por Bernal, (2016); Bryman, (2015); Hernández et al., (2014), Cruz et al., (2014); Tamayo (2012); Hernández (2010); Lerma (2009) y Hurtado (2008). Se utilizó la técnica de la encuesta, mediante un cuestionario estructurado por nueve (9) ítems, para valorar gerencia estratégica, mediante los indicadores: formulación, ejecución y evaluación de estrategias, con escala ordinal de respuestas, (MDA): muy de acuerdo, (DA): de acuerdo, (I): indeciso, (ED): en desacuerdo, (MED): muy en desacuerdo, calificándose desde cinco (5) al uno (1) los enunciados positivos y del uno (1) al cinco (5) los enunciados negativos. De acuerdo al proceso de levantamiento de información en las unidades de análisis, después de aplicar el cuestionario sobre la población estudiada los datos fueron codificados y tabulados empleando el programa SPSS, versión 2.0, procesados e interpretados estadísticamente, utilizando la técnica de análisis descriptivo, de varianza y regresión lineal, con la correspondiente discusión teórica de los resultados, permitiendo llegar a las conclusiones.

Los datos recolectados, previo al análisis exploratorio de los mismos, (Curtosis, asimetría, prueba de Kolmogorov-Smirnof y Caja y Bigotes) y demostrado el cumplimiento de condicionantes, se estableció el estatus y preponderancia de las dimensiones analizadas por la varianza (ANOVA) con la prueba de medias 
(Tukey), para el análisis de los resultados se utilizó un baremo de categorización (ver tabla 2). La validez del instrumento, se sometió a revisión de diez (10) expertos en metodología y gerencia en las organizaciones, aplicándose una prueba piloto en un número de sujetos similares a la población, utilizándose la fórmula de confiabilidad Alfa de Cronbach, dando como resultado un $0,91^{r}$, indicando que el instrumento es altamente confiable.

Tabla 2: Baremo de categorización de la media aritmética estableciendo el comportamiento de la variable gerencia estratégica

\begin{tabular}{|l|l|}
\hline Rango de puntaje & Categorización \\
\hline $1-1,79$ & Pésimo. \\
\hline $1,80-2,59$ & Moderadamente pésimo. \\
\hline $2,6-3,39$ & Moderado. \\
\hline $3,4-4,19$ & Moderadamente óptimo. \\
\hline $4,2-5$ & Óptimo. \\
\hline
\end{tabular}

Los criterios de selección de las unidades de análisis: Carbones del Guasare, S.A de Venezuela; el Complejo Carbonífero de Cerrejón en Colombia y Mina Invierno de Santiago de Chile, obedecen por su similar: (a) naturaleza y razón social al pertenecer al sector minas, (b) aportes al producto interior bruto, (c) capital suscrito, (d) total de activos y pasivos declarados a la nación, (e) cantidad de colaboradores vinculados fijos, (f) tamaño de las empresas y (g) modelo y estructura de gerencias adscritas a la vice presidencia. La población de estudio, compuesta por cincuenta y cinco (55) gerentes, mayores de edad, personal fijo, cargo gerencial, indiferentemente de su estado civil, de cada vice presidencia: financiera, sostenibilidad y asuntos públicos, operaciones, financiera y comercial. La metodología se centró mediante: (a) la selección del tema, revisión bibliográfica, a fin de estructurar planteamiento del problema, referentes teóricos, justificación y objetivo; (b) elaboración de la metodología; (c) construcción del instrumento, sometido a prueba de expertos con el fin de precisar la validez del mismo y elaborar la versión final para la posterior aplicación a la población de estudio; (d) tratamiento estadístico de análisis y discusión de los resultados; (e) y obtención de conclusiones.

\section{RESULTADOS Y DISCUSIÓN}

El análisis de la gerencia estratégica para la gestión de personas del sector minero de Venezuela, Colombia y Chile, es realizado por dos vías: (1) estimar el nivel de consideración de los gerentes que conformaron la población sobre el estado de las etapas de la gerencia estratégica: formulación, ejecución y evaluación de estrategias, y (2) determinar la regresión que mide la incidencia de cada uno de estos indicadores sobre la gestión de personas. En la Tabla 3, (ns) =no significativa, $\left({ }^{*}\right)=$ significativa, $\left({ }^{* *}\right)=$ Altamente significativa, y las letras iguales entre las medias de un aspecto es equivalente a (ns).

En la tabla 3 se muestran los resultados de la primera vía, evidenciando que no existen diferencias significativas sobre la percepción del estado de estas etapas $(F=0,175 ; p \leq 0.84)$. Las medias de 3.06, 3,06 y 2,99 de la evaluación, formulación y ejecución de estrategias respectivamente, en conformidad con el baremo de la tabla 2 son indicativos de su aplicación moderada. Ante estos hallazgos, Fayol (1971) y Serna (2008), en sus teorías manifiestan que los indicadores de la gerencia estratégica se deben aplicar en la organización equitativa e igualmente, por tal motivo es coherente que los resultados muestren la inexistencia de diferencias importantes entre estas etapas, aun cuando se considera como una oportunidad de mejora ya que pudieses ser catalogada como moderadamente óptimo u óptimo, impactando sistémicamente las acciones ejecutadas para el mejoramiento del talento humano. Estos resultados son similares a las investigaciones efectuadas por Mendoza (2015), Fuentes y Cordón (2016), Armstrong (2016) y Chiavenato y Sapiro (2017), los cuales manifiestan que la gerencia estratégica para la gestión de personas, permite sistematizar información cualitativa y cuantitativamente para la toma de decisiones efectivas con criterios y análisis pertinentes. Al comparar los estudios de Dirani (2018), Fred (2018), McDonnell et al., (2017) indican que la variable de estudio genera valor agregado a la cadena socio productiva empresarial, por tal motivo se hace perentorio fortalecer dichas estrategias.

En la tabla 4 (a) es la variable dependiente: gerencia estratégica, muestra los resultados al aplicar la regresión lineal múltiple sobre la ponderación de los indicadores de la variable dependiente: gestión estratégica para la gestión de personas, a mayor valor absoluto del coeficiente mayor es el peso (importancia), en este sentido la ejecución de estrategias es la más importante con el coeficiente 0.511 , 
seguido de la formulación con 0.458 , colocándose de último la evaluación con 0.380 . Estos datos coinciden con las investigaciones de Fred (2018), Tekin y Konina (2018), Doktoralina y Apollo (2019), y Susur et al., (2019), donde manifiestan que la ejecución de estrategias, guarda un valor importante en la gerencia estratégica, sin desmeritar los esfuerzos al ejecutar y evaluar la estrategia.

Tabla 3: ANOVA para las medias de los indicadores de la gerencia estratégica

\begin{tabular}{|c|c|c|c|c|}
\hline Variable & Indicadores de la gerencia estratégica & Media & $F$ & $p$ \\
\hline \multirow[t]{3}{*}{ Gerencia estratégica. } & Formulación de estrategias. & $\begin{array}{c}3.06 \\
\text { (a) }\end{array}$ & \multirow[t]{3}{*}{0.175} & \multirow[t]{3}{*}{$\begin{array}{l}0.84 \\
\text { (ns) }\end{array}$} \\
\hline & Ejecución de estrategias. & $\begin{array}{l}3.06 \\
\text { (a) }\end{array}$ & & \\
\hline & Evaluación de estrategias. & $\begin{array}{l}2.99 \\
\text { (a) }\end{array}$ & & \\
\hline
\end{tabular}

Según investigaciones realizadas por Rao et al., (2018) y Jessica et al., (2018), la gerencia estratégica para la gestión de personas es un desafío clave que enfrentan hoy las organizaciones, desarrollando líderes con talento estratégico, debido a la escasez del capital humano en entornos que son a la vez dinámicos, competitivos y agresivos, donde el ser vivo prevalece por el potencial de sus actitudes y aptitudes entre generaciones, constituyéndose la efectividad laboral como el valor único diferenciador, con escasa trasferencia de conocimiento que limitan el cierre de brechas de aprendizaje en áreas medulares de la organización.

En este mismo orden de ideas, se determinan especificidades de aplicación empresarial y táctica dentro de la organización y límites de su estrategia (Tekin y Konina, 2018; Villalobos y Ramírez 2018), potenciando el desempeño del colaborador, contribuyendo al producto interno bruto (PIB), impacto las mejores prácticas laborales (Doktoralina y Apollo, 2019), integra la (i) articulación de las expectativas y visiones, (ii) construcción de las redes sociales y (iii) actividades de aprendizaje (Susur et al., 2019). Esto con miras a la búsqueda de un entendimiento de cuáles serán futuros talentos, para alinear sus propósitos estratégicos, (Dirani y Nafukho, 2018).

El estudio muestra que la gerencia estratégica para la gestión de personas potencia una ventaja competitiva sostenible "ejecución de estrategias" como rasgo distintivo en el mercado, convirtiéndose el colaborador en generador de valor estratégico por sus competencias, referenciando características cautivadoras, siendo el mecanismo de vinculación de los demás recursos en la empresa, estableciéndose como un sistema de dirección que responda a las exigencias del entorno. Propone, desarrolla y evalúa decisiones multidisciplinarias, con objetivos empresariales cónsonos a la realidad, logrando el éxito organizacional. Analizando el entorno, ventajas competitivas y aprovechamiento de las oportunidades para minimizar las amenazas, ofrece a la empresa indicadores de logros y control mediante cuadros de mandos de gestión, identificando procesos y elementos críticos para fortalecerlos dentro de esta, siendo coherentes con los principios corporativos, apoyando a la estructura y modelo del talento humano.

Al mismo tiempo, se condiciona una nueva tendencia emergente para dirigir a las personas y vincularse a los nuevos retos de las redes empresariales, clúster y stakeholder, adaptadas a las dinámicas globales del mundo actual. Donde se promueva en la organización indicadores a favor de la estrategia empresarial, dirigidas a implementar las mejores prácticas específicas de gestión humana, generando efectos positivosparticipativas en el desempeño del talento humano, propiciando organizaciones felices, con igualdad de género, tendencias verdes, a lo natural, emergente, liderentes, innovador, capacidad de aprendizaje, técnico, profesional y sistémico, apoyadas con las variables responsabilidad social y gestión de conocimiento, donde se garantice la permeancia en el mercado.

Finalmente, contar con la gerencia estratégica para la gestión de personas en el sector minero permite en equipos de trabajo de alto desempeño articular la mejora continua, y facilita la trazabilidad de los objetivos a consolidar mediados por los principios corporativos, de acuerdo a las necesidades organización-persona que requiera el sector real, propiciando la sinergia colaborador-empresa, redundando en prácticas innovadoras de trabajo, bajo un enfoque incluyente, resiliente, responsable, liderente, adaptativo, dignificador, dinámico, participativo, sistémico, generador de valor agregado, aprendible, tecnológico, relacional y resolutivo, donde todos forman parte del resultado final sustentable, indiferentemente de sus roles, perfiles o competencias, dejando a un lado el pretérito paradigma de la centralización, inequidad, desigualdad y autocracia. 
Tabla 4: Análisis de regresión lineal múltiple: estimación de la gerencia estratégica para la gestión de personas

\begin{tabular}{|c|c|c|c|c|c|c|}
\hline \multirow{2}{*}{\multicolumn{2}{|c|}{ Modelo }} & \multicolumn{2}{|c|}{$\begin{array}{l}\text { Coeficientes no } \\
\text { estandarizados }\end{array}$} & \multirow{2}{*}{$\begin{array}{c}\begin{array}{c}\text { Coeficientes } \\
\text { estandarizados }\end{array} \\
\text { Beta }\end{array}$} & \multirow[b]{2}{*}{$t$} & \multirow[b]{2}{*}{ Sig. } \\
\hline & & $B$ & $\begin{array}{c}\text { Error } \\
\text { estándar }\end{array}$ & & & \\
\hline \multirow[t]{4}{*}{1} & (Constante) & 1,359 &, 176 & & 7,738 & ,000 \\
\hline & Formulación de estrategias. & ,176 & ,033 & ,458 & 5,344 & ,000 \\
\hline & Ejecución de estrategias. & 218 & 035 &, 511 & 6,299 &, 000 \\
\hline & Evaluación de estrategias. & 156 & ,035 & 380 & 4,431 & ,000 \\
\hline
\end{tabular}

\section{CONCLUSIONES}

De acuerdo a los resultados y discusión del estudio, se puede extraer que: (1) Entre las etapas de la gerencia estratégica no existen diferencias significativas, considerándose moderada. Al aplicar la regresión lineal múltiple sobre la ponderación de los indicadores de la gerencia estratégica para la gestión de personas, la ejecución de estrategias fue la más importante, seguido de la formulación y evaluación, lo que ofrece un referente contextual para considerar tomas de decisiones pertinentes ante estos hallazgos, fortaleciendo focos críticos como es el de la formulación y evaluación de estrategias, en busca de su optimización.

(2) Los datos reportados en la investigación colocan de manifiesto que la formulación de estrategia, permite hacer un diagnóstico actual y deseado de la organización, mediante actividades formales e informales, identificando necesidades y requerimiento, para formular objetivos coherentes a su realidad. Respecto a la ejecución, son acciones que generan procesos competitivos, considerando integralmente todas las áreas funcionales administrativas y operativas de aplicación, considerándose como la fuerza laboral. La evaluación, analiza externa e internamente, cada etapa de implementación de la estrategia, respondiendo a la dinámica del entorno, originando decisiones asertivas, alimentando criterios de control para administrar responsablemente todos los recursos, blindando la progresiva gestión organizacional y de cada actor que la constituye, evitando cualquier margen de error.

(3) La literatura en referencia confirma que la gerencia estratégica para la gestión de personas, sistematiza información del presente, identificando fortalezas para utilizarse como elemento distintivo ante sus grupos de interés, aprovechar oportunidades, disminuir debilidades, reducir amenazas en un mercado emergente y competitivo, incursionando en nuevos negocios, mediados por equipos de alto desempeño en áreas medulares, alimentando criterios de control, con miras a los indicadores de logros, donde los actores: redes empresariales, clúster y stakeholder hacen parte de iniciativas de aplicación sistémicas, prácticas y originales, con tendencias verdes y renovables. Por tal motivo, se hace imperante articular sistémicamente al personal con los resultados esperados, establecidos en la formulación, ejecución y evaluación de la estrategia, ya que del talento humano de la organización dependerá la consolidación de lo propuesto.

\section{REFERENCIAS}

Armstrong, M., Armstrong's handbook of strategic human resource management, $6^{\text {a }}$ Ed., 001-280, Kogan Page Limited, Philadelphia USA (2016)

Bernal T.C., Metodología de la Investigación, 4ª Ed., 001-384, Editorial Pearson, Bogotá, Colombia (2016)

Boštjančič, E. y Z. Slana, The Role of Talent Management Comparing Medium-Sized and Large Companies - Major Challenges in Attracting and Retaining Talented Employees. Frontiers in Psychology, doi: 10.3389/fpsyg.2018.01750, 9(1), 1750 (2018)

Bryman, A. y E. Bell, Business Research Methods, 4ª Ed., 001-778, Oxford University Press, Oxford, England (2015)

Cruz, C., S. Olivares y M. González, Metodología de la Investigación, 1ª Ed., 001-249, Grupo Editorial Patria, México DF (2014)

Cuesta S.A., Gestión del Talento Humano y del Conocimiento, 5a Ed., 003-437, Ediciones ECOE, Bogotá, Colombia (2015)

Chiavenato, I. y A. Sapiro, Planeación Estratégica. 3ª Ed., 001-344, McGraw Hill Editorial, España (2017)

Delgado Castillo, H., Administración Estratégica. Un Enfoque Metodológico, 2a Ed., 001-296, Editorial Trillas, S.A. de C.V, México (2011)

Dirani, K.M., Talent Management and Development in the United Arab Emirates. Advances in Developing Human Resources, doi: 10.1177/1523422318803088, 20(4, 1), 479-497 (2018) 
Dirani, K.M. y F.M. Nafukho, Talent Management and Development: Perspectives From Emerging Market Economies. Advances in Developing Human Resources, doi: 10.1177/1523422318803362, 20(4), 383-388 (2018)

Doktoralina, C. M. y Apollo, The Contribution of Strategic Management Accounting in Supply Chain Outcomes and Logistic Firm Profitability. Uncertain Supply Chain Management, doi: 10.5267/j.uscm.2018.10.010, 7(2), 145-156 (2019)

Duran, S. E., J.E. Crissien., J. Virviescas y J. E. García, Estrategias gerenciales para la formación de equipos de trabajos en empresas constructoras del Caribe colombiano, ISSN: 0798 1015, Revista espacios, 38(13), 02-15 (2017)

Fayol, H., Administración Industrial y General., 1ª Ed., 001-070, Editorial Universitaria (Chile) - Obras Ilustradas, Santiago, Chile (1971)

Fred R, D., La Gerencia Estratégica, $1^{a}$ Ed., 001-370, Legis Editores, Bogotá, Colombia (2018)

Fuentes, F.M. y P.E. Cordón, Fundamentos de Dirección y Administración de Empresas, 3ª Ed.,001-272, Ediciones Pirámide (Grupo Anaya, S.A), España (2016)

Hernández, S.R., Metodología de la Investigación, 5 a Ed., 89-101, Editorial McGraw - Hill, México (2010)

Hernández, R.F., C. Fernández y M. Baptista, Metodología de la Investigación, 6a Ed.,001-607, Editorial McGraw - Hill, México (2014)

Hurtado de B.J., Cómo Formular Objetivos de Investigación: un Acercamiento Desde la Investigación Holística, $2^{\mathrm{a}}$ Ed., 01-75, Ediciones Gavilán C.A, Caracas, Venezuela (2008)

Jessica, L., A. Hedayati-Mehdiabadi y otros tres autores, Talent Management Process in Asia: a Multiple Case Study. European Journal of Training and Development, doi: 10.1108/EJTD-04-2018-0035, 42(7, 8), 499-516 (2018)

León, S.G., Nuevos Enfoques para la Gestión Estratégica de la I+D e Innovación en las Universidades, ISSN: 0034592X, Revista de Educación,382(355), 83-108 (2011)

Lerma, G.H., Metodología de la Investigación: Propuesta, Anteproyecto y Proyecto, 5ª Ed., 001-190, Ecoe Ediciones, Bogotá, Colombia (2009)

Ling, F.Y., Y. Ning., Y.H. Chang y Z. Zhang, Human Resource Management Practices to Improve Project Managers' Job Satisfaction. Engineering, Construction and Architectural Management, doi: 10.1108/ECAM-02-2017-0030, 25(5), 654$669(2018)$

McDonnell, A., D. G. Collings., K. Mellahi y R. Schuler, Talent management: A systematic review and future prospects. European J. International Management, doi: 10.1504/EJIM.2017.081253, 11(1), 86-128 (2017)

Mendoza, J. M., Decisiones Estratégicas Macroadministración, $1^{\text {a }}$ Ed., 001-330, Editorial Universidad del Norte. Barranquilla, Colombia (2015)

Meyer, K.E. y K.R. Xin, Managing Talent in Emerging Economy Multinationals: Integrating Strategic Management and Human Resource Management. International Journal of Human Resource Management, doi: 10.1080/09585192.2017.1336362, 29(11), 1827-1855 (2018)

Pérez, G., E. Eades, y J. Wilson, Managing human resources in the Latin American context: the case of Chile. The International Journal of Human Resource Management, doi: 10.1080/09585192.2011.639547, 23(15), $3133-3150$ (2012)

Ramírez Molina, R.I. y A. Hugueth, Modelo de Comunicación Productiva para las Organizaciones de Salud Pública en Venezuela, ISSN: 1012-1587, Revista Opción, 33(83), 305-335 (2017)

Ramírez Molina, R.I., J.V. Villalobos y B. Herrera, Proceso de talento humano en la gestión estratégica, ISSN: $1012-$ 1587, Revista Opción,34(18), 2076-2101 (2018)

Ramírez Molina, R.I., C. Espindola., G. Ruíz y A. Hugueth, Gestión del Talento Humano: Análisis desde el Enfoque Estratégico. Información Tecnológica. 30(6), en prensa (2019)

Rao, K.S., Ch. Sahyaja., B.P. Reddy y A. Chakravarthy, Role of a Leader in Strategic Talent Management - A study in Techmahindra, ISSN: 0976-6316, International Journal of Civil Engineering and Technology, 9(2), 247-254 (2018)

Serna, H., Gerencia Estratégica, $1^{a}$ Ed., 001-420, 3 R Editores, Bogotá, Colombia (2008)

Susur, E., A. Hidalgo y D. Chiaroni, A Strategic Niche Management Perspective on Transitions To Eco-Industrial Park Development: a Systematic Review of Case Studies. Resources, Conservation and Recycling, doi: 10.1016/j.resconrec.2018.06.002, 140(1), 338-359 (2019)

Tamayo,M., Técnicas de la Investigación, 3ª Ed.,001-175, Editorial Panapo S. A, México DF (2012)

Tekin, A. V. y O. V. Konina, The role of Information and Communication Technologies in the Process of Strategic Management of Entrepreneurial Structures Activities: The Budget and Financial Aspect. Advances in Intelligent Systems and Computing, doi: 10.1007/978-3-319-90835-9_32, 726(1), 269-278 (2018)

Villalobos, J. V. y R.I. Ramírez, El derecho a la autobiografía: dimensión ius-filosófica desde la perspectiva de H. Arendt y P. Ricoeur, ISSN: 1012-1587, Revista Opción,34(18), 1012-1587 (2018)

Wheelen, T. L, J. D. Hunger., A. N. Hoffman y C. E. Bamford, Strategic management and business policy : globalization, innovation, and sustainability, $15^{\mathrm{a}}$ Ed.,001-178, Edition Pearson, México DF (2018) 